Pacific Journal of Mathematic 


\title{
ON FINITE GROUPS CONTAINING A CCT-SUBGROUP WITH A CYCLIC SYLOW SUBGROUP
}

\author{
MARCEL HerzoG
}

Let $G$ be a finite group containing a CCT-subgroup $M$. M is called a $C C T$-subgroup of $G$ if $M$ contains the centralizer in $G$ of each of its nonunit elements and it is also a trivial-intersection subset of $G$. In this paper the $p$-blocks of characters of $G$ of full defect are described in detail, under the additional assumption that the Sylow $p$-subgroups of $M$ are cyclic and nontrivial. This information yields, under the same conditions, a detailed characterization of the nonexceptional (with respect to $M$ ) irreducible characters of $G$. As an application, it is shown that if $G$ is also perfect, its order is less than $q m\left(m^{2}+3 m+2\right) / 2$, where $m$ is the order of $M$ and $q m$ is the order of $N_{G}(M)$, and $N_{G}(M) \neq M, G$, then $G$ is isomorphic either to $\operatorname{PSL}(2, p)$, $m=p>3$, or to $\operatorname{PSL}(2, m-1), m-1=2^{b}, b>1$. These results generalize those of $R$. Brauer, dealing with the case $m=p$.

We proceed with a precise statement of the results. Quite recently, E. C. Dade [4] obtained a very detailed information about blocks with cyclic defect groups. Using his results, we will prove the following

TheOREM 1. Let $G$ be a finite group of order $g$ and suppose that $G$ contains a subgroup $M$ of order $m$, satisfying the following conditions:

(i) for all $h \in M^{*}, C_{G}(h) \subseteq M$

(ii) a Sylow p-subgroup $P$ of $M$ is cyclic of order $p^{a}, a \geqq 1$

(iii) $q=\left[N_{G}(M): M\right]>1, g>q m$.

Then the nonexceptional (with respect to $M$ ) irreducible characters of $G$, nonvanishing on $M^{*}$, are of one of the following two types:

(I) $P_{i}(i=1, \cdots, y)-P_{i}(1)=r_{i} m+1, P_{i}(h)=1$ for all $h \in M^{\sharp}$

(II) $Q_{i}(i=1, \cdots, q-y)-Q_{i}(1)=s_{i} m-1, Q_{i}(h)=-1$ for all $h \in M^{\#}$

where $1 \leqq y \leqq q$ and $r_{i}, s_{i}$ are nonnegative integers.

The assumptions of Theorem 1 imply that $M$ is a nilpotent Hall subgroup of $G$, which is a trivial-intersection-subset of $G$. It follows, hence, that the Sylow $p$-subgroups of $G$ are cyclic, of order $p^{a}$. The situation described in Theorem 1 is similar to that which was summarized by $R$. Brauer in [1, pp. 59-60], for the case that $M$ satisfies condition (i) and $m=p$.

Theorem 1 follows quite easily from the following proposition, 
which is of independent interest. Since we are to distinguish between the (irreducible) exceptional characters of $G$ with respect to $M$ (see W. Feit [5]) and those with respect to the cyclic $p$-group $P$ (in the sense of E. C. Dade [4]), the latter ones will be called $p$-exceptional. An irreducible character is $p$-nonexceptional if it is not $p$-exceptional.

Proposition. Let $G$ and $M$ satisfy the assumptions of Theorem 1. Let $t$ be the number of conjugate classes of $G$ meeting $M^{\sharp}$ nontrivially. Then the $p$-blocks of $G$ are listed and described below:

( I ) $d$ blocks of defect 0 , each containing one ordinary irreducible character and one modular irreducible character.

(II) One block of defect $a$, containing $q$ modular irreducible characters, $q$-nonexceptional characters and $\left(p^{\alpha}-1\right) / q p$-exceptional characters.

(III) $\left(t q+1-p^{a}\right) / p^{a} q$ blocks of defect $a$, each containing one modular irreducible character, one $p$-nonexceptional character and $p^{a}-1$ $p$-exceptional characters.

As an application of Theorem 1, we will prove the following generalization of the main result of $R$. Brauer in [1]. Theorem 1 is also needed in a forthcoming paper.

THEOREM 2. Let $G$ be a finite group of order $g$ and assume that $G=G^{\prime}$. Suppose that $G$ contains a subgroup $M$ of order $m$, satisfying conditions (i)-(iii) of Theorem 1.

Then $g=q m(n m+1)$, where $n$ is a positive integer, and one of the following statements holds:

( I ) $n=\left(m u w+u^{2}+u+w\right) /(u+1)$

where $u$ and $w$ are positive integers.

(II) $\quad n=1, m=p, q=(p-1) / 2, G \cong P S L(2, p), p>3$.

(III) $n=(m-3) / 2, q=2, m=2^{b}+1>3, G \cong P S L(2, m-1)$.

As an immediate corollary we get:

Corollary. Let $G$ and $M$ satisfy the assumptions of Theorem 2. If $n<(m+3) / 2$, then $G$ is a simple group either of type (II) or of type (III).

The arguments applied in the proof of Lemma 2.2 are quite similar to those used by $R$. Brauer in his proof of Theorem 7 in [1]. However, for the sake of completeness, the proof is given in full detail.

Most of our notation is standard. If $G$ is a group, then $G^{\#}$ and $1_{G}$ denote, respectively, the nonunit elements of $G$ and its principal 
irreducible character. If $p$ and $q$ are rational integers, then $(p, q)$ denotes the greatest common divisor of $p$ and $q$, and $p \mid q$ means: $p$ divides $q$. If $u, e, h$ are elements of the group $G$, then $n(u)$ is the order of the centralizer of $u$ in $G$ and $c_{u e h}$ is the coefficient of the conjugate class of $h$ in the product of the conjugate classes of $u$ and $e$.

1. Proof of the Proposition and of Theorem 1. In this proof by blocks we mean $p$-blocks. Since $q>1, M$ is a nilpotent group and $P \subseteq Z(M)$. It follows, in view of assumptions (i) and (ii), that $G$ has blocks of defect 0 and $a$ only. The blocks of defect 0 are well known to be of type (I)(see [3], Th. 86.3). Therefore it remains to show that the blocks of defect $a$ are either of type (II) or of type (III), and their number is correct.

If $m=p$, then the Proposition and Theorem 1 follow by Brauer [1,pp.59-60]. In that case $G$ has only one block of defect $a$, as required. Therefore, from now on, we will assume that $m>p$. Since $N_{G}(M)$ is a Frobenius group and $P$ is cyclic, it follows that $q \leqq p-1$ and $q<\sqrt{m-1}$.

As $P$ is cyclic, the blocks $B_{1}, \cdots, B_{u}$ of defect $a$ were described by Dade [4]. Each $B_{i}$ contains $e_{i}$ modular irreducible characters, $e_{i}$ $p$-nonexceptional characters and $\left(p^{a}-1\right) / e_{i} p$-exceptional characters, where $e_{i}$ is an integer dividing $q$.

Let $d^{\prime}$ be the number of the irreducible characters of $G$ vanishing on $M^{\sharp}$. We will show that $d^{\prime}=d$. Let $X$ be an irreducible character of $G$ vanishing on $M^{\sharp}$. Then $m \mid X(1)$, and since $m$ is divisible by the full $p$-part of $|G|, X$ has defect 0 . Thus $d^{\prime} \leqq d$. On the other hand, $G$ and $M$ satisfy the assumptions of Hypothesis $A$ in [6]. By [6], Lemma 3.1.d, $c=0$. Hence, according to the formulas following Hypothesis A, the degrees of the irreducible characters of $G$, nonvanishing on $M^{*}$, are of one of the following forms:

$$
z(f m+q \varepsilon), r m+s
$$

where $f$ and $r$ are nonnegative integers, $\varepsilon= \pm 1, s$ is the value taken on $M^{\sharp}$ by the corresponding nonexceptional character, hence a nonzero integer, and $z$ is a degree of an irreducible character of $M$. Since $P$ is a normal abelian subgroup of $M$, it follows by Ito (see [3], Corollary $53.18)$ that $z$ divides $[M: P]$, hence $(z, p)=1$. As also $(q, p)=1$, it follows that no character of degree $z(\mathrm{fm}+q \varepsilon)$ belongs to a block of defect 0 . Since by the definition of $T$ and by [6], Lemma 3.1.b $s \leqq T \leqq q$, it follows that $(s, p)=1$ and no character of degree $r m+s$ belongs to a block of defect 0 . Consequently, $d \leqq d^{\prime}$ and the equality follows.

It is well known, that the number of the modular irreducible 
characters of $G$ is equal to the number of (conjugate) classes in $G$ minus the number of $p$-singular classes in $G$ (see [3], Th. 83.5). In view of the nilpotency of $M$, the number of the $p$-singular classes in $M$ is $\left(p^{a}-1\right)(t q+1) / p^{a}$, and the corresponding number for $G$ is $\left(p^{q}-1\right)(t q+1) / q p^{a}$. Let $b$ be the number of the nonexceptional characters of $G$, nonvanishing on $M^{*}$. Then, $t$ being the number of the exceptional characters of $G$, the number of the conjugate classes in $G$ is $t+b+d$. It follows from the above mentioned equality, remarks and the results of Dade that:

$$
\sum e_{i}+d=t+b+d-\left(p^{a}-1\right)(t q+1) / q p^{a}
$$

where the summation is over $i=1, \cdots, u$. The index of summation $i$ will run over these values throughout this proof. It follows that:

$$
\sum e_{i}=t+b-\left(p^{a}-1\right)(t q+1) / q p^{a} .
$$

Since the number of the (ordinary) irreducible characters of $G$ is equal to the number of the conjugate classes in $G$, we get, using once more Dade's results, that after subtraction $d$ from both sides:

$$
\left(p^{a}-1\right) \sum\left(1 / e_{i}\right)+\sum e_{i}=t+b .
$$

Equations (1) and (2) yield:

$$
\begin{gathered}
t=p^{a} \sum\left(1 / e_{i}\right)-(1 / q) \\
b=\sum e_{i}-\sum\left(1 / e_{i}\right)+(1 / q) .
\end{gathered}
$$

It is also well known, that the number of blocks of defect $a$ is equal to the number of $p$-regular conjugate classes of $G$ of defect $a$ (see [3], Th. 86.10). Thus:

$$
u=t-\left(p^{a}-1\right)(t q+1) / q p^{a}+1=\left(t q+1-p^{a}\right) / p^{a} q+1 .
$$

Finally let $q=e_{i} f_{i}$. It follows from (3) and (5) that:

$$
\begin{gathered}
u=(1 / q) \sum f_{i}-(1 / q)+1 \\
\sum f_{i}=u q+1-q .
\end{gathered}
$$

Suppose that $f_{i} \neq q$. Then $f_{i} \leqq q / 2$ and it follows from (6) that at most one $f_{i}$, say $f_{1}$, is unequal to $q$. Hence:

$$
f_{1}+(u-1) q=(u-1) q+1
$$

and consequently:

$$
f_{1}=1, e_{1}=q, f_{2}=\cdots=f_{u}=q, e_{2}=\cdots=e_{u}=1 .
$$

The Proposition follows from (5), (7) and the results of Dade. 
Equations (4) and (7) immediately yield $b=q$. Thus there are $q$ nonexceptional characters nonvanishing on $M^{\sharp}$ and therefore, in view of [6], Lemma 3.1.b, they are either of type (I) or of type (II). As the principal character is of type (I), $y \geqq 1$. This completes the proof of Theorem 1.

2. Proof of Theorem 2. By [6], Theorem 2.3, $N_{G}(M)=Q M$, where $Q \cap M=1$ and $g=q m(n m+1)$. Since $q \neq 1, M$ is a nilpotent group with a cyclic Sylow subgroup, hence $Q$ is abelian. As $G=G^{\prime}$, $n \neq 0$.

If $m=p$, then the theorem holds by Brauer ([1], Th. 10). Therefore, from now on, we will assume that $n$ does not satisfy (I), $m>p$ and will prove that then $G$ satisfies (III). Since $P$ is cyclic, it follows that $1<q<\sqrt{m}-1$ and $q \leqq p-1$.

The groups $G$ and $M$ now satisfy the assumptions of Hypothesis $\mathrm{A}$ in [6]. Therefore it follows from Theorem 3.1 there that $c=0$, and by Thorem 1 of this paper, $c_{i}= \pm 1$. Consequently, taking into account the formulas preceding Theorem 3.1 in [6], the degrees of the nonprincipal irreducible characters of $G$, nonvanishing on $M^{\sharp}$, are:

$$
\text { the exceptional characters: } z(b m+q \varepsilon)=(z / v)[(b v+\varepsilon) m-\varepsilon]
$$

the nonexceptional characters: $u m \pm 1$

where $u$ is a positive integer, $b$ is a nonnegative integer, $\varepsilon= \pm 1$, $v=(m-1) / q$ and $z$ is a degree of an irreducible character of $M$. By Ito ([3], Corollary 53.18) $z$ divides $[M: P]$, hence $z<v$.

We will proceed with a series of lemmas.

Lemma 2.1. $G$ is a simple group and $m$ is an odd integer.

Proof. Let $K$ be a normal complement of $N_{G}(M)$ in $G$. Then $K$ is nilpotent and $G$ is solvable, a contradiction. Therefore $N_{G}(M)$ has no normal complement in $G$.

Let now $K$ be a nontrivial, proper normal subgroup of $G$. Then by [6], Theorem 2.3, the order of $K$ is either of the form $w m+1$, where $w m+1$ divides $n m+1$ and $n=y w m+y+w$ for some nonnegative integer $y$, or it is of the form $q_{0} m(n m+1)$, where $q_{0}$ divides $q$. Suppose that the first case occurs. As shown above, $w \neq n$, and hence $y$ is a positive integer. Let $x=(w+1) y$; then

$$
n=\left(x w m+x+w^{2}+w\right) /(w+1),
$$

which has been assumed not to be the case. On the other hand, the order of $K$ could not be $q_{0} m(n m+1)$, since that would force the exis- 
tence of a nonprincipal irreducible character of $G$ of degree less than $q$, which is not the case. Thus $G$ is a simple group.

If $m$ is even, then it follows from Suzuki ([8]), Theorem 1) and the fact that $q \neq 1$, that $M$ is a Sylow 2-group of $G$. Hence $p=2$ and consequently $q=1$, a contradiction. It follows that $m$ is odd, and the proof of the lemma is complete.

LEMma 2.2. The degrees of the nonprincipal irreducible characters of $G$, nonvanishing on $M^{\sharp}$, are:

the exceptional characters: $\quad z(n m+1) / v$

the nonexceptional characters: $m-1$ or $n m+1$ where $z$ and $v$ are as described above.

Moreover, $\varepsilon=-1$ and $v \mid n+1$.

Proof. In view of (8), it suffices to show that if

$$
u m+1 \mid(m-1)(n m+1)
$$

then $u=n$, if $u m-1 \mid(m-1)(n m+1)$ and $u m-1 \neq(m-1)(n m+1)$ then $u=1$, and no irreducible character of $G$ of degree $z(m-1) / v=z q$ exists. Indeed, by (8) each character in question has order of the form $k(u m+\delta)$, where $k$ is either 1 or $z / v$ and $\delta= \pm 1$. If $\delta=1$ then $u=n$, as required. If $\delta=-1$, then either $u m-1=(m-1)(n m+1)$ or $u=1$. If $u=1$, then $k=1$ and the corresponding character is not an exceptional one. If $u m-1=(m-1)(n m+1)$, then:

$$
k^{2}(u m-1)^{2} \geqq(m-1)^{2}(n m+1)^{2} / v^{2}>q m(n m+1)
$$

a contradiction. Thus if $k=z / v$ then $\delta=1$ and $u=n$; it follows also that $\varepsilon=-1$ and $v$ divides $n m+1-n(m-1)=n+1$.

Let $u m+1=d e$, where $d \mid m-1$ and $e \mid n m+1$. Suppose that $n<u$. Then $d \mid u+1$ and $e \mid u-n$. Hence $(u+1)(u-n)=w(u m+1)$, where $w$ is a positive integer and:

$$
\begin{gathered}
u+1 \mid w(m-1), u \leqq w m-w-1 \\
u^{2}=u(w m+n-1)+w+n, u>w m+n-1
\end{gathered}
$$

a contradiction. If on the other hand, $n>u$ then $d \mid u+1$ and $e \mid n-u$. It follows that $(u+1)(n-u)=w(u m+1)$, where $w$ is a positive integer, and $n$ is of form (I), a contradiction. Thus $u=n$.

Let $u m-1=d e$, where $d \mid m-1$ and $e \mid n m+1$. Suppose that $u>1$. Then $d \mid u-1$ and $e \mid n+u$. Hence:

$$
(u-1)(n+u)=w(u m-1)
$$

where $w$ is a positive integer, and considering this equation as a 
quadratic equation for $u$, we get that the second root $u^{\prime}=(w-n) / u$ is an integer. Since for $u=1$ the left hand side of (9) vanishes, while the right hand side is positive, it follows that $u^{\prime} \leqq 0$. Let $u^{\prime}=-u^{\prime \prime}$, and suppose first that $u^{\prime \prime}=0$. Then $w=n$, and (9) yields:

$$
u=n(m-1)+1, u m-1=(n m+1)(m-1) .
$$

Therefore if $u m-1 \neq(n m+1)(m-1)$ then $u^{\prime \prime}$ is a positive integer and $-u^{\prime \prime}$ satisfies equation (9). Consequently, $n$ satisfies (I), which is not the case. Thus $u=1$.

Finally assume that $x=z q$ is a degree of an irreducible character of $G$. As $z$ is a degree of an irreducible character of $M^{\#}$, it is well known that $z^{2} \leqq[M: Z(M)] \leqq m / 2 p$. It follows that

$$
x=z q \leqq \sqrt{m / 2 p} \sqrt{m}-1<(m-1) / 2 .
$$

But then, since $G$ is simple, the character of degree $x$ satisfies the assumptions of Theorem 4.2 [7]. This is a contradiction, since the group $G$ does not satisfy any of the conclusions of that theorem. Thus no irreducible character of degree $z q$ exists, and the proof of the lemma is complete.

LEMMA 2.3.

$$
g=(m-1) m[(q-1) m-q]
$$

and no nonexceptional character of $G$ is of degree $n m+1$.

Proof. Let $h \in M^{\sharp}$ and let $y$ have the same meaning as in Theorem 1. Then it follows from Lemma 2.2, Theorem 1 and from the summation formulas of $\S 3$ in [6] that:

$$
\begin{aligned}
0 & =\sum X(1) X(h) \\
& =1+(f m-q)(-\varepsilon)+(y-1)(n m+1)-(q-y)(m-1)
\end{aligned}
$$

where the summation ranges over all the irreducible characters of $G$ and $f=(n+1) / v, f m-q=(m n+1) / v$. After cancellation, we get:

$$
q=f+y(n+1)-n=(n+1) / v+y(n+1)-n .
$$

Suppose that $y>1$; then, as $v \leqq n+1$ :

$$
q>(n+1) / v+n+1>v, m-1=q v<q^{2}
$$

in contradiction to our assumptions. Therefore $y=1$ and $n=(q-1) v-1$. Consequently, there are no nonexceptional characters of degree $n m+1$, and:

$$
g=q m[(q-1) v m-(m-1)]=(m-1) m[(q-1) m-q] .
$$


LEMMA 2.4.

$$
q=2 .
$$

If $u$ is an involution of $N_{G}(M)$, then $n(u)=m-1$.

Proof. By Lemma 2.3,

$$
g=(m-1) m r
$$

where $r=(q-1) m-q$. It is easy to see that the three factors are prime to each other.

Let $u, h$ and $e$ be elements of $G^{*}$, whose orders divide $m-1, m$ and $r$, respectively. Let $X, R$ and $D$ be: an exceptional character of $G$, a nonprincipal nonexceptional character of $G$ nonvanishing on $M^{*}$ and an irreducible character of $G$ vanishing on $M^{*}$, respectively. Let $s$ be a prime divisor of the order of $u$. Then $R$, being of degree $m-1$, belongs to an $s$-block of defect 0 , and consequently $R(u)=0$ (see [3], Th. 86.3). Similarly, $X(e)=0$. We, therefore, get the following character table:

$\begin{array}{ccccc} & 1 & u & h & e \\ 1_{G} & 1 & 1 & 1 & 1 \\ X & z(n m+1) / v & & & 0 \\ R & m-1 & 0 & -1 & \\ D & a m & & 0 & \end{array}$

By the well known formula for $c_{u e h}$ it follows that:

$$
c_{u e h}=g / n(u) n(e), \quad n(u) n(e) \mid(m-1) r .
$$

Since $e$ is an arbitrary element of order dividing $r$, it follows that $n(u) \mid m-1$.

As $m$ is odd, $m-1$ is even. Let now $u$ be an involution of $G$, and let $C$ be the conjugate class of $G$ containing $u$, of order $c$. Then:

$$
c \geqq g /(m-1)>g / m-1
$$

and by [2], Theorem (4K), and the trivial intersection property of $M$, $q$ is even.

Let, finally $u$ be an involution belonging to $N_{G}(M)$. Then it follows from [2], Theorem (4J), that $n(u) \geqq m-1$. Since $n(u) \mid m-1$, $n(u)=m-1$. The same theorem also yields:

$$
g=(m-1) m(m-q) \text {. }
$$

Thus, in view of Lemma $2.3, q=2$ and the proof of the lemma is complete. 
Theorem 2 now follows from Lemma 2.4 and a result of Suzuki [9]. Suzuki has shown, that if Lemma 2.4 holds, then $G$ is of type (III).

\section{REFERENCES}

1. R. Brauer, On permutation groups of prime degree and related classes of groups, Ann. of Math. 44 (1943), 55-79.

2. R. Brauer, and K. A. Fowler, On groups of even order, Ann. of Math. 62 (1955), 565-583.

3. C. W. Curtis, and I. Reiner, Representation Theory of Finite Groups and Associative Algebras, Interscience Publishers, 1962.

4. E. C. Dade, Blocks with cyclic defect groups, Ann. of Math. 84 (1966), 20-48.

5. W. Feit, On a class of doubly transitive permutation groups, Illinois J. Math. 4 (1960), 170-186.

6. M. Herzog, On finite groups which contain a Frobenius subgroups, J. Algebra 6 (1967), 192-221.

7. H. S. Leonard, Jr., On finite groups which contain a Frobenius factor group, Illinois J. Math. 9 (1965), 47-58.

8. M. Suzuki, Two characteristic properties of (ZT)-groups, Osaka Math. J. 15 (1963), $143-150$.

9. _ - An abelian subgroup which is the centralizer of its non-identity elements, Lecture, Midwestern Conference on Group Theory and Number Theory, Columbus, Ohio, 3.30-4.2., 1966.

Received April 24, 1967.

UNIVERSITY OF ILLINOIS

URBANA, ILLINOIS 



\title{
PACIFIC JOURNAL OF MATHEMATICS
}

\author{
EDITORS
}

\section{H. ROYDEN}

Stanford University

Stanford, California

\author{
J. P. JANS \\ University of Washington \\ Seattle, Washington 98105
}

\section{J. DugundJI}

Department of Mathematics

University of Southern California

Los Angeles, California 90007

\section{RICHARD ARENS}

University of California

Los Angeles, California 90024

\section{ASSOCIATE EDITORS}
E. F. BECKENBACH
B. H. NEUMANN
F. WOLF
K. YOSIDA

\section{SUPPORTING INSTITUTIONS}

\author{
UNIVERSITY OF BRITISH COLUMBIA \\ CALIFORNIA INSTITUTE OF TECHNOLOGY \\ UNIVERSITY OF CALIFORNIA \\ MONTANA STATE UNIVERSITY \\ UNIVERSITY OF NEVADA \\ NEW MEXICO STATE UNIVERSITY \\ OREGON STATE UNIVERSITY \\ UNIVERSITY OF OREGON \\ OSAKA UNIVERSITY \\ UNIVERSITY OF SOUTHERN CALIFORNIA
}

\author{
STANFORD UNIVERSITY \\ UNIVERSITY OF TOKYO \\ UNIVERSITY OF UTAH \\ WASHINGTON STATE UNIVERSITY \\ UNIVERSITY OF WASHINGTON \\ AMERICAN MATHEMATICAL SOCIETY \\ CHEVRON RESEARCH CORPORATION \\ TRW SYSTEMS \\ NAVAL WEAPONS CENTER
}

Mathematical papers intended for publication in the Pacific Journal of Mathematics should be in typed form or offset-reproduced, double spaced with large margins. Underline Greek letters in red, German in green, and script in blue. The first paragraph or two must be capable of being used separately as a synopsis of the entire paper. It should not contain references to the bibliography. Manuscripts, in duplicate if possible, may be sent to any one of the four editors. All other communications to the editors should be addressed to the managing editor, Richard Arens, University of California, Los Angeles, California 90024.

Each author of each article receives 50 reprints free of charge; additional copies may be obtained at cost in multiples of 50 .

The Pacific Journal of Mathematics is published monthly. Effective with Volume 16 the price per volume (3 numbers) is $\$ 8.00$; single issues, $\$ 3.00$. Special price for current issues to individual faculty members of supporting institutions and to individual members of the American Mathematical Society: $\$ 4.00$ per volume; single issues $\$ 1.50$. Back numbers are available.

Subscriptions, orders for back numbers, and changes of address should be sent to Pacific Journal of Mathematics, 103 Highland Boulevard, Berkeley 8, California.

Printed at Kokusai Bunken Insatsusha (International Academic Printing Co., Ltd.), 7-17, Fujimi 2-chome, Chiyoda-ku, Tokyo, Japan.

PUBLISHED BY PACIFIC JOURNAL OF MATHEMATICS, A NON-PROFIT CORPORATION

The Supporting Institutions listed above contribute to the cost of publication of this Journal, but they are not owners of publishers and have no responsibility for its content or policies. 


\section{Pacific Journal of Mathematics}

\section{Vol. 25, No. $3 \quad$ November, 1968}

Philip Marshall Anselone and Theodore Windle Palmer, Collectively

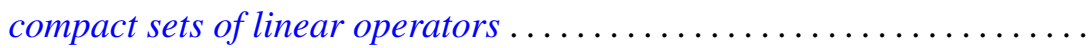

Philip Marshall Anselone and Theodore Windle Palmer, Spectral analysis of collectively compact, strongly convergent operator sequences.........

Edward A. Bender, Characteristic polynomials of symmetric matrices...... 433

Robert Morgan Brooks, The structure space of a commutative locally convex

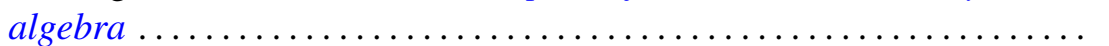

Jacob Feldman and Frederick Paul Greenleaf, Existence of Borel

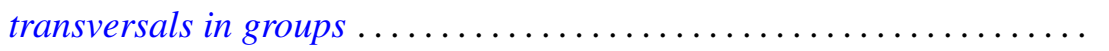

Thomas Muirhead Flett, Mean values of power series 463

Richard Vernon Fuller, Relations among continuous and various

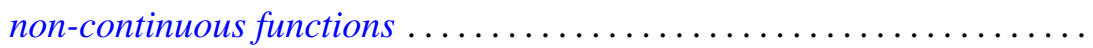

Philip Hartman, Convex sets and the bounded slope condition ............

Marcel Herzog, On finite groups containing a CCT-subgroup with a cyclic Sylow subgroup .

James Secord Howland, On the essential spectrum of Schroedinger

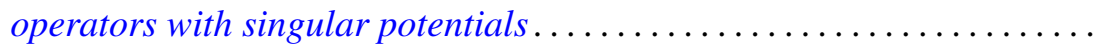

Thomas William Hungerford, On the structure of principal ideal rings .....

Paul Joseph Kelly and Ernst Gabor Straus, Curvature in Hilbert geometries. II mpati Madhusudana Rao, Linear functionals on Orlicz spaces:

Malempati Madhusudana Rao, Linear functionals on Orli
$\quad$ General theory............................
Stanley F. Robinson, Theorems on Brewer sums.......
Ralph Tyrrell Rockafellar, A general correspondence betw

Malempati Madhusudana Rao, Linear functiona
$\quad$ General theory.........................
Stanley F. Robinson, Theorems on Brewer sums
Ralph Tyrrell Rockafellar, A general corresponden

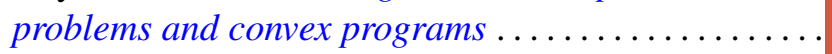

Richard Benjamin Sher, Defining subsets of $E^{3}$ by cubes.

Howard Jacob Weiner, Invariant measures and Cesàro summability... 